\title{
THE EFFECTS OF FINITE RESOLUTION ON RADAR IMAGES OF FRACTAL PROFILES
}

\author{
Gerardo Di Martino, Antonio Iodice, Daniele Riccio, Giuseppe Ruello \\ Dipartimento di Ingegneria Elettronica e delle Telecomunicazioni, \\ Università di Napoli “Federico II", Via Claudio 21, 80125 Napoli, Italy \\ E-mail: \{gerardo.dimartino, iodice, daniele.riccio, ruello\}@unina.it
}

\begin{abstract}
In this paper a first step toward a complete model of the fractal imaging process is taken: for the sake of simplicity the mathematical details are here provided for a fractal profile with topological dimension equal to one. In particular, we show how the signal backscattered from a fractal profile modeled as a fractional Brownian motion $(\mathrm{fBm})$ stochastic process is strictly linked to an associated fractional Gaussian noise (fGn) process. We compute in closed form the power density spectrum of the received signal in the simplified hypothesis of a linear dependence of the backscattered signal on the profile derivative process. Our results apply to physical $\mathrm{fBm}$ processes, as dictated by the low-pass filtering introduced by both the incident electromagnetic field wavelength and the finite sensor resolution.

In the last section a numerical study of the abovementioned is also provided.
\end{abstract}

Index Terms - Fractals, microwave scattering

\section{INTRODUCTION}

In the last years, new generation sensors are supplying a huge amount of high resolution data relative to almost any part of the world. The absence of accurate analytical models connecting the image to the observed scene parameters is one of the main reasons limiting the extraction of information from these data. In fact, this type of direct modeling is of key importance not only to better understand radar images relevant to natural scenes, but could be of great support in the retrieving of geophysical parameters from this kind of images.

In this paper we focus on the mathematical and numerical modeling of the radar imaging process of natural surfaces. We use here a fractal description for the imaged surface: indeed, in literature it is widely recognized that such a model describes the geometry of natural surfaces better than any other one based on Euclidean geometry [1], [2]. Among fractal models, we use the fractional Brownian motion ( $\mathrm{fBm}$ ) model [1], [2], because it provides one of the best mathematical characterizations of natural surfaces using a minimum set of independent fractal parameters. However, here we focus on the (Euclidean) onedimensional problem, highlighting our novel results without hampering the mathematical details. As a matter of fact, isotropy and depolarization issues would occur in the extension to the (Euclidean) two-dimensional problem.

This kind of direct modeling is not widespread in literature: the main weakness of the existing works is that none of them puts forward a completely fractal framework. Conversely, in this work the fractal modeling involves both the geometric and the electromagnetic issues.

First of all, assuming the validity of the linear model originally proposed by Pentland [3], we obtain an expression for the power density spectrum of the image, thus providing a closed form solution for the small slope regime. In particular, we show that the image relative to an $\mathrm{fBm}$ profile falling in this regime is strictly connected to the associated fGn [4], which describes the derivative process of the profile. These results are obtained introducing a smoothed version of the original, nondifferentiable profile: the rationale of this model allows taking into account the effects of finite sensor resolution on the final image.

Finally, our analytical results are numerically validated through the fractal processing chain recently presented by the authors [5]. Note that in this case no simplifying hypothesis, such as the abovementioned linearity one, is assumed and use is made of a fractal SPM scattering model [2] to compute the signal backscattered from the $\mathrm{fBm}$ profile.

\section{FRACTAL MODELS}

In this paper we use an $\mathrm{fBm}$ stochastic process to model the profile. It can be defined through the statistics of its increments. In fact, a stochastic process $z(x)$ is an $\mathrm{fBm}$ profile if, for every $x$ and $x^{\prime}$, it satisfies the following relation: 


$$
\operatorname{Pr}\left\{z(x)-z\left(x^{\prime}\right)<\bar{\zeta}\right\}=\frac{1}{\sqrt{2 \pi} s \tau^{H}} \int_{-\infty}^{\bar{\zeta}} \exp \left(-\frac{\zeta^{2}}{2 s^{2} \tau^{2 H}}\right) d \zeta
$$

where $\tau=\left|x-x^{\prime}\right|, H$ is the Hurst coefficient $(0<H<1)$, related to the fractal dimension $D$, and $s\left[\mathrm{~m}^{(1-H)}\right]$ is the standard deviation ..of the profile increments at unitary distance.

The structure function $V(\tau)$ (whose plot is termed the variogram) is defined as the mean square increment of elevation points placed at distance $\tau$ and for an fBm profile can be evaluated in terms of the parameters $H$ and $s$ as:

$$
V(\tau)=s^{2} \tau^{2 H}
$$

It has been demonstrated [1], [2] that the power density spectrum $S(k)$ of an isotropic $\mathrm{fBm}$ one-dimensional process exhibits appropriate power-law behaviors provided by:

$$
S(k)=s^{2} \frac{\pi H}{\cos (\pi H)} \frac{1}{\Gamma(1-2 H)}|k|^{-(2 H+1)},
$$

$\Gamma(\cdot)$ being the Gamma function.

Note that both the structure function (2.2) and the power spectrum (2.3) of an $\mathrm{fBm}$ profile exhibit a linear behavior in a $\log -\log$ plane, allowing the use of linear regression techniques for the retrieving of the fractal parameters of the profile from measured data.

As stated in Section I, in the analytical part of our work we assume the model proposed by Pentland [3] to be valid. The hypothesis underlying to this model is that the observed profile exhibits a small slope regime, at least at sensor resolution scale: if this is the case, we can assume a linear relation between the backscattered signal intensity and the profile derivative. Hence, we need an expression for the derivative process of the $\mathrm{fBm}$ profile, which is also known as fractional Gaussian noise (fGn). Obviously, this process has to be handled with care because of the fBm lack of derivative: a way to solve this problem is to smooth the original process by the means of an adequate kernel of support $[0, \varepsilon]$.

Thanks to the choice of an effective kernel, the autocorrelation function of the derivative process $z^{\prime}(x ; \mathcal{E})$ can be simply evaluated. Furthermore, after this filtering, the processes $z(x ; \mathcal{E})$ and $z^{\prime}(x ; \mathcal{E})$ are stationary. Hence, from the autocorrelation function we can readily obtain the structure function of $z^{\prime}(x ; \varepsilon)$.

The next step is the evaluation of the power density spectrum of the process: being it stationary we can compute this spectrum via the Wiener-Kintchine theorem. Eventually, we obtain the following result:

$$
S_{z^{\prime}}(k ; \varepsilon)=2 s^{2} \varepsilon^{-2} \Gamma(1+2 H) \sin (\pi H)(1-\cos (k \varepsilon))|k|^{-(2 H+1)}
$$

and in the limit of $\varepsilon<<\frac{2 \pi}{k}$ :

$$
S_{z^{\prime}}(k)=s^{2} \Gamma(1+2 H) \sin (\pi H)|k|^{-(2 H-1)} .
$$

From the results presented above we can draw two important conclusions. First of all, from (2.5) follows that the fGn process exhibits the interesting property of being an asymptotically power law process, with exponent $1-2 H$. This is equivalent to say that upon differentiation the Hurst parameter of the process is decreased by one, at least asymptotically. The other key conclusion, is that this process is not a fractal one, both looking at (2.4), and this is quite obvious, and looking at (2.5): in fact, as we mentioned above, for this process $H \notin[0,1]$ and, therefore, it is not possible to define an Hausdorff-Besicovitch fractional dimension for this class of processes [1].

\section{IMAGING MODEL}

The relation between the images of natural profiles and the fGn process presented in the previous section is here shown.

As we said in the first section of this paper, in our analytical computations we assume Pentland model [3] to be valid. According to that model, the imaging process can be seen as a system whose input is the surface profile, and whose output is the radar image, which depends on the derivative of the observed profile. As a matter of fact, this means that we are assuming a MacLaurin first order series expansion of the image intensity function with respect to the derivative of the considered profile.

If this is the case, the image intensity is linearly related to the derivative of the profile and the results obtained in the previous section can be easily exploited. In particular, note that the smoothing effect obtained in the previous section by the means of the smoothing kernel can be related here to the effects of finite sensor resolution. In fact, system resolution has a low pass filtering effect on the profile: we can assume, without loss of generality, that the support of the abovementioned smoothing kernel is $\Delta x$, where $\Delta x$ is the resolution cell dimension. A block diagram of this kind of imaging process is shown in Fig.1.

If $a_{1}$ is the coefficient relative to the linear term of the abovementioned MacLaurin expansion, we can write the power density spectrum $S_{I}$ of the image intensity as:

$$
S_{I}(k ; \Delta x)=a_{1}^{2} S_{z^{\prime}}(k ; \Delta x) .
$$




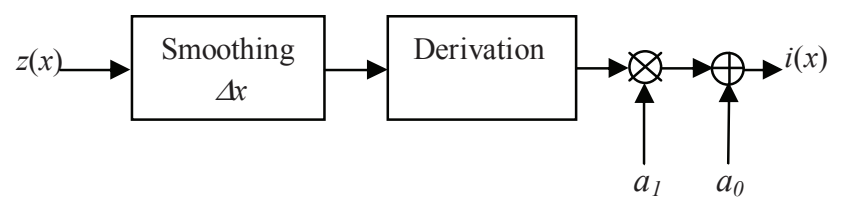

Fig. 1 Block diagram of the linear imaging process: $i(x)$ is the image intensity; $a_{1}$ and $a_{0}$ are the constant term and the coefficient of the linear term of the MacLaurin series expansion of $i(x)$, respectively.

The $\log -\log$ plot relative to (3.1), compared to that of the profile, as described by (2.3), is presented in Fig. 1. The values of the parameters of interest are shown in Table I.

It is worth noting that our asymptotic result is analogous to that obtained by Pentland [3], due to the fact that in his work he assumed $\varepsilon=0$. Note that this is one of the main limitations of Pentland analysis, together with his heuristic use of the Lambertian scattering behavior. In fact, at least at microwave frequencies, the scattering is definitely not Lambertian-like and has been demonstrated that the use of adequate fractal scattering models, based for example on the Physical Optics or the Small Perturbation Method [2], [6], provides a huge increase in the accuracy of the backscattered signal evaluation. Anyway, as mentioned above it is possible to find this kind of weaknesses in almost all the works published on this subject (see, as an example, [7] and [8]).

Some observations on the profile fractal parameters retrieving are now in order. As previously mentioned, the fractal parameters of an $\mathrm{fBm}$ profile can be estimated via a simple linear regression on measured height data, where use can be made of the expressions presented in (2.2) and in (2.3). Conversely, when the retrieving of these parameters from the image intensity is under concern equation (3.1) testifies that a simple estimation technique can no longer be used. However, in the asymptotic limit of (2.5) the estimation of the fractal parameters can be still performed via the standard technique.

\section{NUMERICAL RESULTS}

In the present section an experimental framework, based on effective direct geometric and electromagnetic models, is presented. This framework is essentially based on the fractal processing chain recently presented by the authors [5].

We generated a fractal $\mathrm{fBm}$ profile using the Weierstrass-Mandelbrot (WM) function: under some hypothesis, the WM effectively approximates an $\mathrm{fBm}$ profile [2], [3]. Furthermore, this is a predictable random function and this makes it easier to control the behavior of the profile, acting on its parameters.

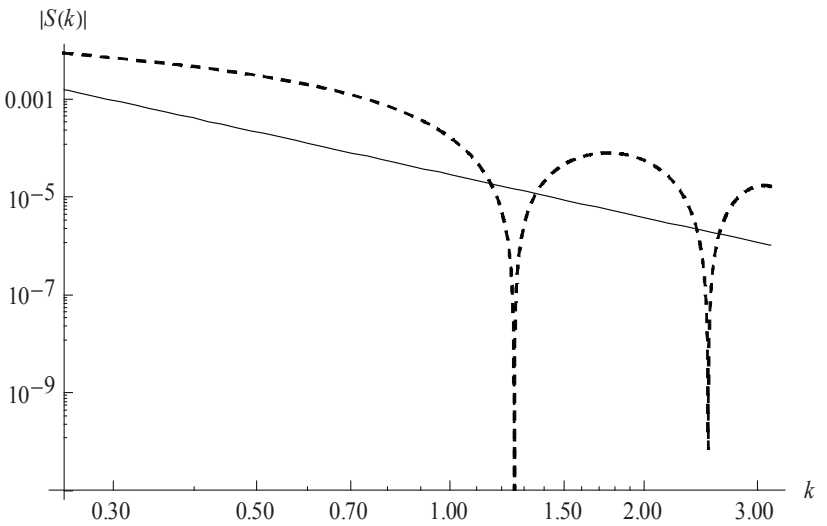

Fig. 2 Power density spectrum of the profile (full line) and of its image.

Table I

Values of the parameters of interest for the figures.

\begin{tabular}{|c|c|c|c|c|}
\hline & $H$ & $s\left[\mathrm{~m}^{1-H}\right]$ & $\Delta x[\mathrm{~m}]$ & $a_{1}$ \\
\hline Fig. 2-3 & 0.95 & 0.01 & 5 & 10 \\
\hline Fig. 4 & 0.55 & 0.01 & 20 & 10 \\
\hline
\end{tabular}

Once the profile was synthesized, we evaluated the backscattered signal via an SPM fractal scattering model [2]. The geometrical model used in this section is based on the assumption that the observed profile shows the same fractal parameters at all the scales of interest: in particular, at scales greater and lower of the resolution one. Note that, if this was not the case, the parameters $H$ and $s$ used in (3.1) would refer to the fractal parameters of the profiles at resolution scale [5].

To validate the results presented in the previous sections, we estimated the power density spectra of the profile and of the backscattered signal. Note that particular care has to be taken in the evaluation of these power law spectra, because they are subject to extreme leakage and high variance problems. Hence, we used a Capon filter to circumvent these problems [9].

In Fig. 3 significant numerical results are presented: the values of the involved parameters are shown in Table I.

Note that the particular choice of the parameters, both those relative to the surface ( $H$ and $s$ ) and to the system $(\Delta x)$, determines a good agreement between theoretical and experimental spectra. If those parameters are changed, so that the linearity hypothesis on which is based this work falls, such a degree of agreement is not reached. As an example, in Fig. 4 we show the same plots of Fig. 3 with different values of the parameters, still shown in Table I. From Fig. 4 it is evident that the fit between the spectrum of the image and the theoretical behavior is not as good as in the previous case. 


\section{CONCLUSIONS}

In this paper we presented the first steps toward a complete analytical model of the imaging process of a fractal surface.

First of all, we provided a closed form analytical solution for the case of a profile satisfying a small slope regime. For this case we have shown that the asymptotic behavior of the power density spectrum of the image intensity is power law, but its exponent is such that this image cannot be said to be fractal.

As for the numerical results, we have shown that, when the parameters of interest, relevant to both the profile (in particular, $H$ ) and the remote sensing system (in particular, the system resolution $\Delta x$ ), are chosen in a way such that the linear model assumed in the analytical computations can be considered satisfied, there is a good agreement between the experimental and analytical results. Conversely, if this is not the case, the goodness of fit between the two behaviors begins to decrease.

The main strength of the presented approach is the use of a completely fractal framework: in fact, the authors are aware that this is the only way to avoid inaccurate results, as is frequently the case in the existing literature on this subject.

\section{REFERENCES}

[1] B.B. Mandelbrot, The Fractal Geometry of Nature. New York: Freeman, 1983.

[2] G. Franceschetti, D. Riccio, Scattering, Natural Surfaces and Fractals. Academic Press, Burlington (MA), USA, 2007.

[3] P. Kube and A. Pentland, "On the imaging of fractal surfaces", IEEE Trans. Pattern Anal. Mach. Intell., vol. 10, no. 5, pp.704707, Sep. 1988.

[4] B. B. Mandelbrot and J. W. Van Ness, "Fractional Brownian motions, fractional noises and applications", SIAM Rev., vol. 10, no.4, pp.422-437, 1968.

[5] G. Di Martino, A. Iodice, D. Riccio, G. Ruello, "A novel approach for disaster monitoring: fractal models and tools", IEEE Trans. Geosci. Remote Sens., vol. 45, no. 6, pp. 1559-1570, June 2007.

[6] G. Franceschetti, 1A. Iodice, D. Riccio, "Fractal models for scattering from natural surfaces", Invited Chapter in Scattering, R. Pike and P. Sabatier, Eds. London, U.K.: Academic, Sep. 2001, pp. 467-485.

[7] G. Korvin, "Is the optical image of a non-lambertian fractal surface fractal?", IEEE Geosci. Remote Sens. Lett., vol. 2, no. 4, pp. 380-383, Oct. 2005.

[8] P. Addesso, S. Marano, R. Restaino, M. Tesauro, "Correlation properties of signals backscattered from fractal profiles", IEEE
Trans. Geosci. Remote Sens., vol. 45, no. 9, pp. 2859-2868, Sep. 2007.

[9] T. Austin, A. W. England, G. H. Wakefield, "Special problems in the estimation of power-law spectra as applied to topographical modeling", IEEE Trans. Geosci. Remote Sens., vol. 32, no. 4, pp. 928-939, July 1994.

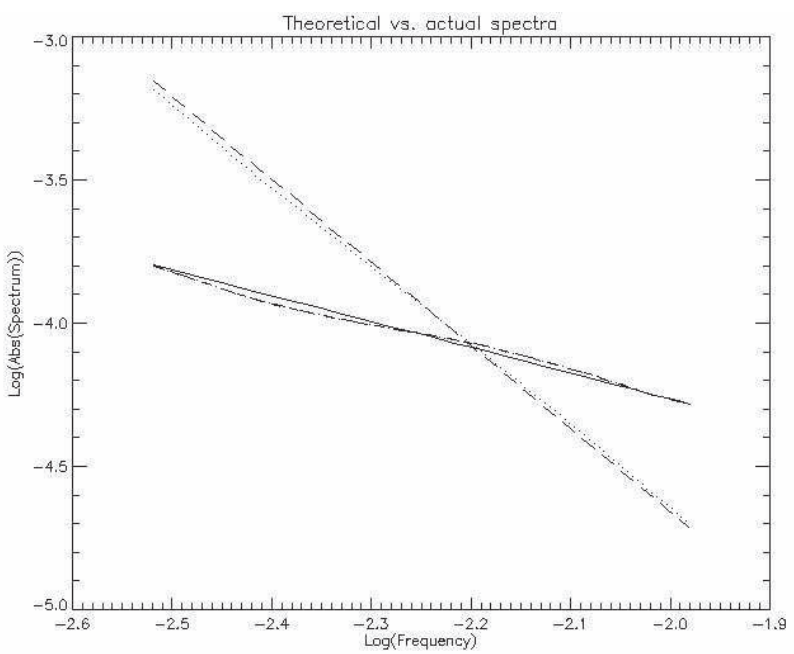

Fig. 3 Theoretical spectra of the surface (long dashed) and of the image (full line) vs. estimated ones (dotted and dash dot dot, respectively).

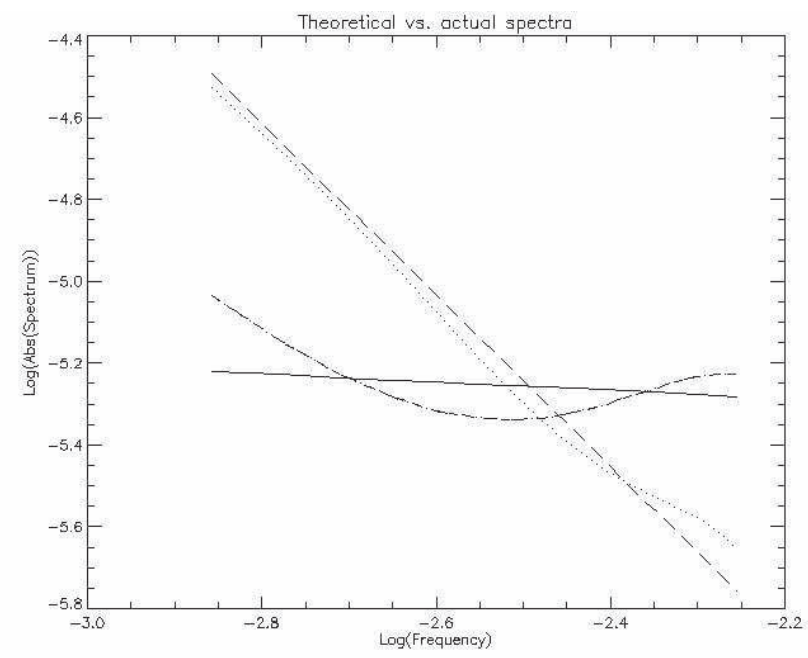

Fig. 4 Theoretical spectra of the surface (long dashed) and of the image (full line) vs. estimated ones (dotted and dash dot dot, respectively). 